\title{
Performance Investigation of Hybrid AC/DC Microgrids during Mode Transitions
}

\author{
Jiannan Liu, Student member, IEEE, M. J. Hossain, Senior Member, IEEE, Md. Shamiur Rahman, Student member, IEEE, \\ Junwei Lu, Senior Member, IEEE \\ Queensland Micro and Nano Technology Centre, Griffith School of Engineering, \\ Griffith University, Australia. \\ johnny.liu@griffithuni.edu.au, j.hossain@griffith.edu.au, mdshamiur.rahman@griffithuni.edu.au, j.lu@griffith.edu.au
}

\begin{abstract}
The hybrid AC/DC microgrid is getting popular in different countries including Australia as it could enhance energy efficiency and system's reliability. This paper investigates the dynamic performance of a hybrid AC/DC microgrid during transition from grid-tied mode to isolated mode and vice versa. As the droop control method allows the system to operate under both grid-connected mode and islanded mode, it is adopted as the main inverter control strategy for observing the system's performance during mode switching. Different characteristics of distribution energy resources (DERs) make it complicated to share power between the DC subgrid and the AC subgrid during islanded mode. Therefore, this paper investigates the performance of a hybrid microgrid under different scenarios which contains various DER combinations. DER units such as PV modules, wind turbines, battery energy storage systems (BESSs), and micro-turbines are utilized for building systems with different structures. Different case studies are carried out in MATLAB/SIMULINK environment based on a real microgrid system which is built in Griffith University Nathan campus for demonstrations. Simulation results show that the microgrid system could perform well and ensure smooth transition with the proposed control strategy described in this paper. Through the comparisons of different scenarios, it can be seen that system which include BESS could show better performance during mode transition. When micro-turbine involves in power supply, fluctuations in AC voltage and power supply can be optimized.
\end{abstract}

Index Terms-Hybrid AC/DC microgrid, wind turbine, PV module, battery energy storage, micro-turbine, droop control.

\section{INTRODUCTION}

With the requirements of energy saving and environmental protection, traditional fossil fuel energy is no longer the first choice in power industries. As an alternative option, the distributed energy resources (DERs) have become the research focus in recent years. DER units include several devices, for example, photovoltaic (PV) units, wind turbines (WTs), micro-turbines (MTs), battery energy storage systems (BESSs) etc. [1] However, devices like PV and WT possess significant challenges due to their intermittent and variable outputs which may cause problems when they are connected to utility grids. The microgrid (MG) concept is proposed to realize the flexible and efficient application of DER units. The microgrid is a small-scale generation and distribution system consisting of DER units, energy storage systems, energy conversion devices, monitoring and protection equipment and loads. It is an autonomous system which is able to achieve high flexibility, operation efficiency, selfcontrollability, self-protection and self-management.

AC microgrid is widely utilized to connect DER units to conventional AC power systems [2]. However, a DC-based microgrid is much easier to control and more economical than an AC microgrid. In recent years, the concept of a hybrid AC/DC microgrid is proposed to combine the advantages of both AC and DC microgrids [3]. DER units and BESS which only output DC power can be connected to a common DC bus through DC/DC converters in which case they constitute a DC microgrid to supply power to DC loads. This DC microgrid can be treated as a unique power supply which needs to be connected to the AC bus through power electronic inverters. A hybrid microgrid combines the advantages of both the AC microgrid and the DC microgrid which makes it more practical and economical to use.

A hybrid microgrid has three operation modes, namely islanded mode, grid-connected mode and transition mode between the two modes mentioned above [4]. During the transition from grid-connected to islanded mode, proper voltage and frequency should be maintained for islanded loads to balance the power generation and demand. A control strategy should be carefully designed in case momentary interruption occurs [5]. When the microgrid is reconnected to the utility grid, synchronization of the microgrid voltage and frequency to the utility grid is required [6]. The switching transient in the behaviours of a hybrid microgrid varies for different DER units involved since each unit has its own special effect on the inverter output.

In [7], a typical hybrid microgrid model is proposed and its performance under grid-connected mode is observed. A P$\mathrm{Q}$ control method and current control strategy are used for the AC and DC side inverters respectively. The designed system in [7] performs well however the islanded case is not considered. In [8, 9], a droop control method is applied in 
hybrid microgrids so that the $\mathrm{AC}$ side frequency and the $\mathrm{DC}$ side voltage can remain stable in both grid-tied and island conditions. An improved control method is introduced in [10] to reduce circulating current and power-sharing deviation between AC and DC microgrids. Authors in [11] concentrate on the issues related to seamless mode transition in a hybrid microgrid. In order to achieve the seamless transfer, the system minimizes the switching items between the PQ control mode and the V/f control mode. However, the utilization of two control strategies makes the controller structure more complex. The hybrid microgrid enables the combination of an AC microgrid and DC microgrid which makes it more suitable for practical applications. Several experimental test systems have been built and tested in different countries and results show that the hybrid microgrids could maintain a good operating performance under both grid-connected and island modes [12, 13]. However, their performance analyze during mode transitions are paid little attention.

In this paper, performances of a hybrid AC-DC coupled microgrid with different structures during the transition mode are investigated. Several case studies are carried out and the response of frequency, AC voltage, DC voltage, active power and reactive power under different scenarios are compared. Inclusions of the dynamics of DER units can assist in analysing the performance of a hybrid microgrid accurately and identifying any critical parameters that can degrade its dynamic performance during switching period. This study will assist in designing control for a hybrid microgrid with different combinations of DER units for its reliable and stable operations.

The rest of the paper is organized as follows. Section II describes the hybrid AC/DC microgrid system configuration as well as the simulation model. Section III discusses the control strategies for the DC/AC inverter which combines the $\mathrm{AC}$ subgrid and the DC subgrid. Case studies covering PV only; PV with battery; WT with battery; PV with WT; PV and WT with battery; PV, WT and MT with battery are presented in section IV. Section V summarizes the conclusions of this study.

\section{SYSTEM DESCRIPTION}

The simulation model used in this paper is mainly based on the real microgrid system configuration developed at Griffith University, Australia, which is shown in Fig. 1. The system comprises of low voltage distribution systems with DER units. Practical loads are from three buildings i.e. N44, N05 and N74 on Nathan campus. New loads such as EVs will also be included in the near future. The N44 building utilizes $15.5 \mathrm{~kW}$ PV panels to achieve peak demand shaving. Wind turbine and battery are planned for installation to reduce power consumption. For the N05 building, an EV charging station is planned for vehicle-to-grid implementation. In the N74 building a micro-turbine is used as a back-up power supply to provide an uninterruptible power supply (UPS) system. In addition, a smart DC/AC inverter (d-Statcom) is also included, which allows for the charging and discharging of the battery energy storage system (BESS). For EVs, the charging station is still in its planning stage, and a simplified model (which mainly includes N44 building and N74 building components) is simulated in MATLAB/SIMULINK environment.

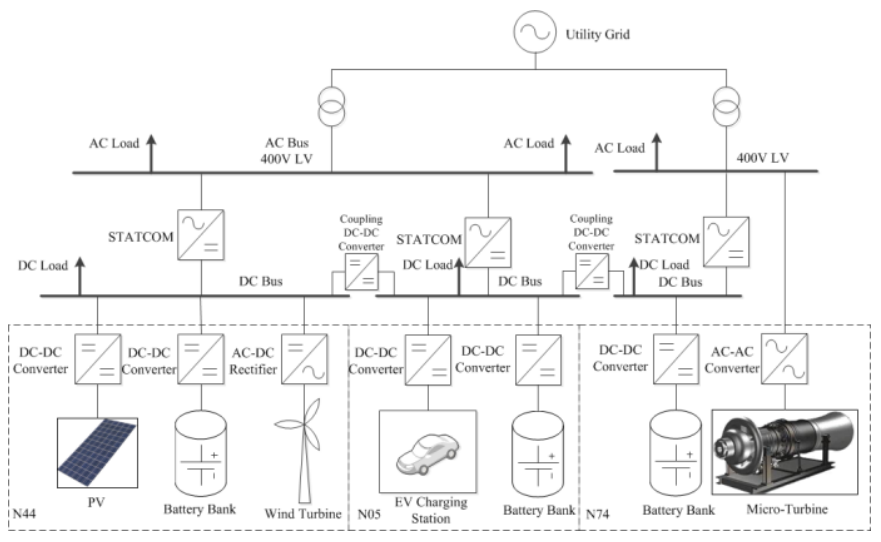

Fig. 1 Hybrid microgrid system configuration of Griffith University

The complete simulation model comprises of two parts: a DC microgrid and an AC microgrid. On the DC side, a PV module and wind turbine generation system with maximum power point tracking (MPPT) controllers and integrated boost converters are included. A lithium-ion battery bank model is also connected to the DC bus to simulate the real BESS. The DC microgrid utilizes a bidirectional inverter to connect to the AC bus which allows power to flow to and from AC and DC sides. In reality, the micro-turbine generation module has an inner converter installed, with which it connects to the AC bus directly. The utility grid is also linked to the $\mathrm{AC}$ bus through an isolated transformer, which forms an AC microgrid with the MT generation system. A fully developed simulation model configuration is shown in Fig. 2.

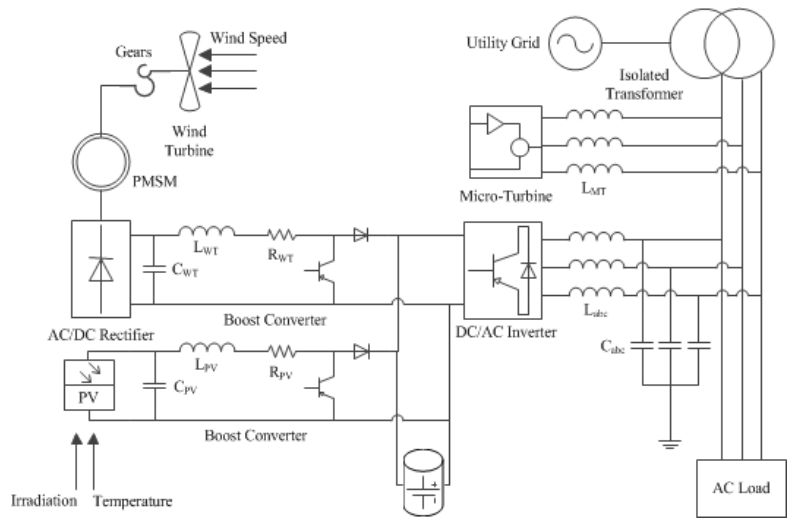

Fig. 2 Simulation model of the hybrid microgrid

\section{MODELLING OF HYBRID MICROGRIDS}

In the hybrid microgrids, an interlinking inverter is necessary to connect the DC bus with the AC bus. Therefore, a robust control strategy for the inverter is important for power exchange between the two subgrids. In addition, according to the Griffith University microgrid system, a single-shaft micro-turbine (with its own converter) is adopted as a back-up power supply. A specialized module for microturbine with a PQ (real and reactive power) control strategy is 
developed in this paper. This section describes the model of the devices under consideration.

\section{A. Interlinking inverter controller}

For inverter-based generation systems, active and reactive power is related to frequency and voltage magnitude respectively. Such relationships (as shown in Fig. 3) lead to the application of the droop control strategy. Droop control can be used to share power among the multiple sources without communication in distribution systems [14]. It allows distribution generators to operate under both grid-connected and islanded modes, which makes it a proper control strategy for the interlinking inverter in this research.

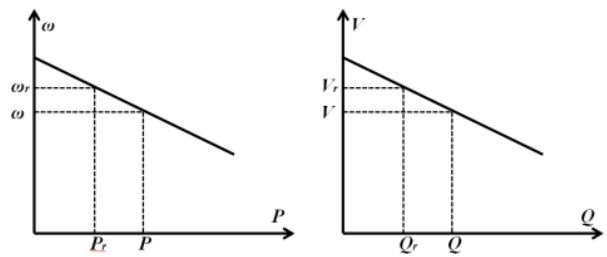

Fig. 3 Frequency and voltage droop characteristic

The inverter controller proposed in this paper contains two subsystems:

1) Power Controller: As frequency is easier to measure, this controller utilizes frequency control instead of phase control. The frequency loop and voltage loop determine the reference voltage and reference phase angle respectively. Active power and reactive power can then be decoupled through a vectorial transformation. The controller used in this paper (shown in Fig. 4) is developed on the basis of the controller proposed in [15].

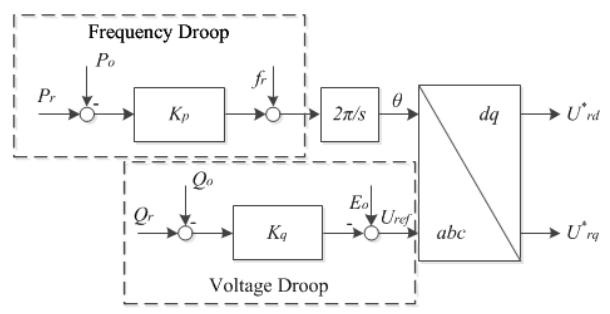

Fig. 4 Block diagram of power controller

2) Voltage-Current Loop Controller: The voltage regulating loop uses load voltage as a control variable. A proportional-integral-derivative (PID) controller is applied to eliminate the tracking errors so that the steady state accuracy can be improved. The output of the voltage loop is transferred into reference current as the input of the current loop. The capacitor current is adopted as a feedback signal. A proportional controller is introduced to enhance the high dynamic response and its steady state error has no effect on the voltage loop accuracy. The capacitor current is then transferred back to voltage component to generate PWM signals [17]. The configuration of the voltage current dual loop controller is shown in Fig. 5.

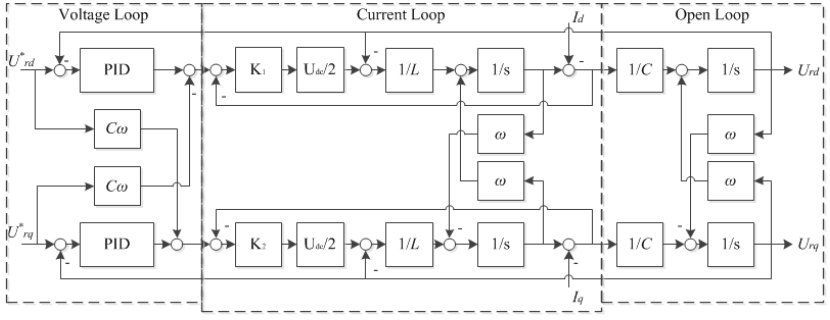

Fig. 5 Block diagram of voltage-current loop controller

\section{B. Micro-Turbine Module}

Based on the Griffith University microgrid system, a single-shaft micro-turbine is adopted as a back-up power supply. The major turbine engine components include a compressor, a recuperator (exhaust gas heat exchanger), a combustor, a turbine, and a generator. Fig. 6 shows a simplified model of single-shaft micro-turbine, in which fast dynamics of the micro-turbine is neglected. Since the recuperator only serves to increase the turbine efficiency, it is not included in the model [16].

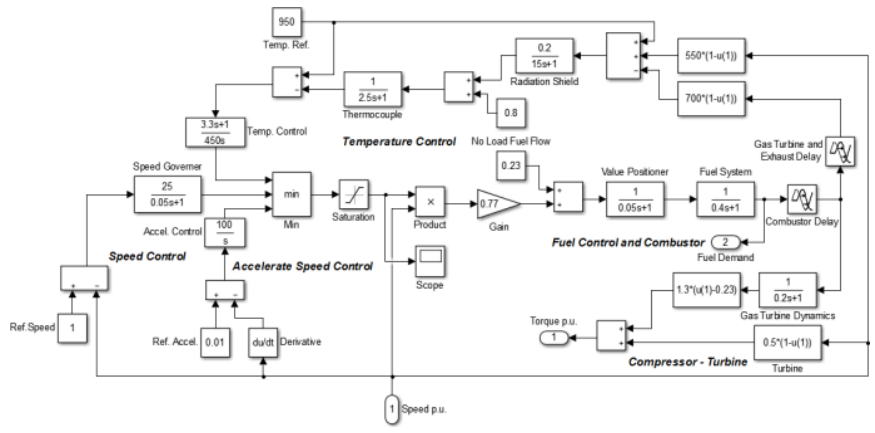

Fig. 6 Simulation model of the micro-turbine

As can be seen, the micro-turbine model mainly consists of five parts: speed control, accelerate speed control, temperature control, fuel control and combustor, and compressor and turbine. Speed control takes effect under variable load conditions. Accelerate speed control is used for avoiding over speeding and temperature control acts as an upper power output limit. The output of all three blocks will be sent to a least value gate which picks the lowest value as the input for the fuel control and combustor block. After optimizing the fuel requirement, the least amount of fuel can be given to the compressor-turbine block. The compressorturbine is a linear and non-dynamic device, which is described by the compressor-turbine block. Output torque follows the linear relationship with fuel flow and can be calculated through this block.

\section{Micro-Turbine Converter Controller}

According to the practical microgrid system configuration, the micro-turbine equipment ready in use includes its own converter installed inside. As a distribution generation resource, the power output of the micro-turbine should be controllable. The converter control strategy proposed in this paper for the micro-turbine adopts the P-Q control method which is shown in Fig. 7. 


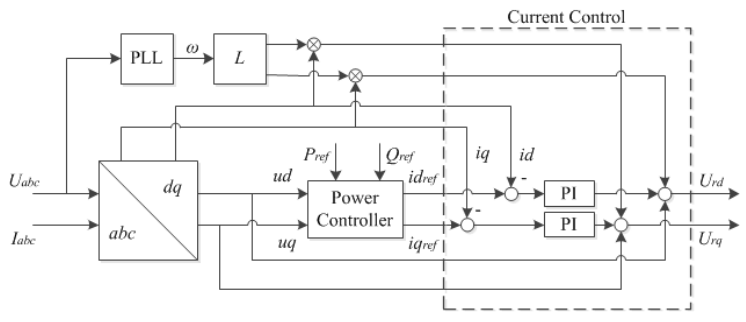

Fig. 7 Block diagram of the micro-turbine converter controller

Through such a control strategy, there is no need for the micro-turbine to consider frequency control and voltage control. The inverter will output active power and reactive power on the basis of the reference value. Similar to the droop control strategy, the P-Q control method also needs to decouple the active power and reactive power by using a power controller to generate reference current. Through current control, the steady state error can be reduced to zero by properly tuning the PI controller.

\section{CASE STUDY}

The main aim of this paper is to study the performance of hybrid microgrids with different configurations of renewable energy sources and distribution energy resources during mode transitions. Different scenarios as mentioned below are simulated and compared based on the system structure presented in Fig. 2:

Case A: a microgrid system which includes PV only.

Case B: a microgrid system which includes PV and battery.

Case $C$ : a microgrid system which includes wind turbine and battery.

Case D: a microgrid system which includes PV and wind turbine.

Case E: a microgrid system which includes PV, wind turbine and battery.

Case F: a hybrid microgrid system which includes PV, wind turbine, battery and micro-turbine.

\section{A. AC Voltage Frequency}

The AC side phase voltage of the microgrid system is set at $230 \mathrm{~V}, 50 \mathrm{~Hz}$ and the total $\mathrm{AC}$ load is $95 \mathrm{~kW}+54 \mathrm{kVar}$. The approximate percentage of power contribution from each DER is shown in the table in Appendix A. The system was initially grid connected and it is intentionally disconnected from the host grid during $4 \sim 7 \mathrm{~s}$. The effect of different system configurations on the frequency response can be observed in Figs. 8 and 9.

During the transition from grid-connected to islanded mode, there is an increase in DER active power output which results in increasing the frequency during 4 4.1s. Although there is an overshoot for a short period of time, the frequency stabilises to $50 \mathrm{~Hz}$ within very short period of time. The microgrid in case A shows the worst performance since it has the largest overshoot and takes longer time to recover. Frequency in case B and case D shows the similar trend which is better than in case A. Frequency in cases C, E and F recovers quickly and smoothly. However, when a microturbine is added in the system, there are little fluctuations in frequency during islanded mode which can be neglected. During the transition from islanded to grid-tied mode, the result shows different scenarios. Cases B, C, E and F show the similar characteristic in which the frequency synchronizes to the grid frequency fluently after a small overshoot occurred. There are more fluctuations in case A which takes more time to stabilise. In case $\mathrm{D}$, another big overshoot occurs during synchronization. It can be concluded that when the PV array produces a higher power output, it is difficult to keep the frequency constant during the transition mode. Since the micro-turbine is connected on the AC bus, its synchronization to the microgrid may affect the frequency under islanded mode. In addition, the battery performs better for frequency synchronization when reconnected to the grid.

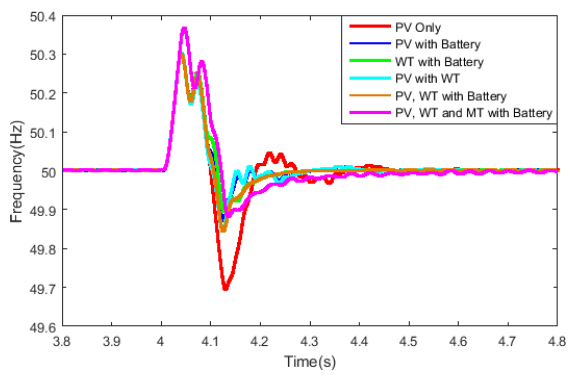

Fig. 8 AC voltage frequency from grid-connected to islanded mode

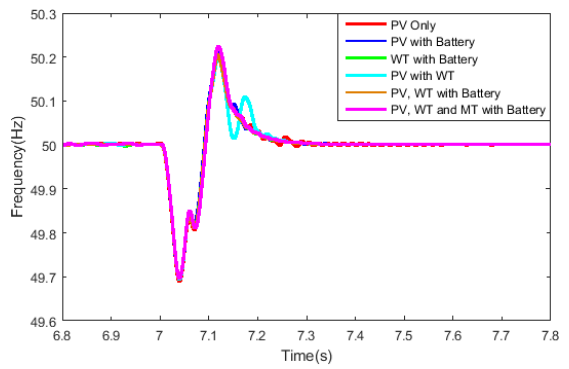

Fig. 9 AC voltage frequency from islanded to grid-connected mode

\section{B. AC Voltage and DC Voltage}

The response of $\mathrm{AC}$ and $\mathrm{DC}$ bus voltage during mode transition are shown in Figs. 10 - 13. Since the power outputs of different cases are not precisely the same, the rms voltage during islanded mode can vary. The voltage response during mode switching is similar in cases A, B, C, D and E. In case $\mathrm{F}$, overshoot of $\mathrm{AC}$ voltage during $4 \sim 4.05 \mathrm{~s}$ is smaller when microgrid disconnected from the grid. Since micro-turbine could offer stable output, it is able to reduce the fluctuations in the output power.

However, the characteristic of DC voltage is significantly different from that of AC voltage. When disconnected from the grid, the utility grid stopped supplying power to loads. Without the support of the main grid, the power output of DER units in microgrid increases to match the AC loads. This is the reason why the AC and DC bus voltage increased during the switching mode. As with frequency, during mode switching from isolated to grid-connected mode, there are more fluctuations in the DC bus voltage which leads to a 
longer recovery time in case A. In addition, it is hard for WT to stabilize its DC bus voltage alone by using uncontrolled rectification. The PV unit is a DC generation which can produce stable DC voltage by using a DC-DC converter. Through the parallel connection with the PV unit, the DC bus voltage of a PV-WT hybrid microgrid can be stable. However with the integration of a WT, a great ripple in DC voltage still happens in case $\mathrm{D}$ during the microgrid reconnection in $7.1 \sim 7.2 \mathrm{~s}$. Furthermore, because the micro-turbine is connected to the AC bus and needed to be synchronized, it takes microgrid longer to regulate $\mathrm{AC}$ side voltage in order to reach in steady-state. In this case, the DC bus voltage also needs more time to become stable.

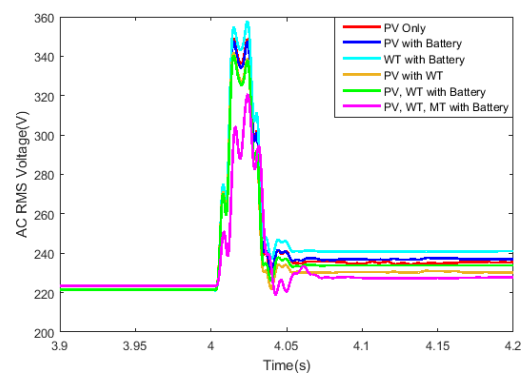

Fig. $10 \mathrm{AC}$ rms voltage from grid-connected to islanded mode

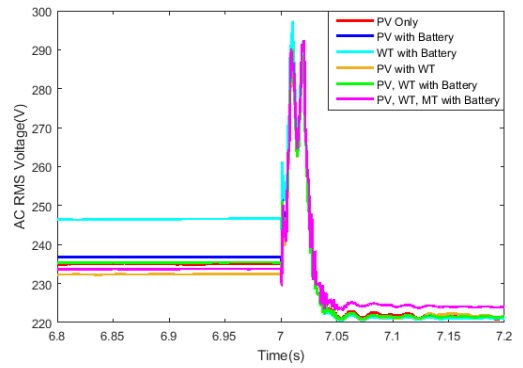

Fig. 11 AC rms voltage from islanded to grid-connected mode

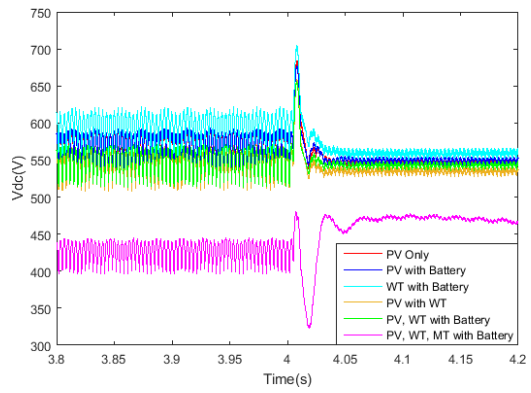

Fig. 12 DC bus voltage from grid-connected to islanded mode

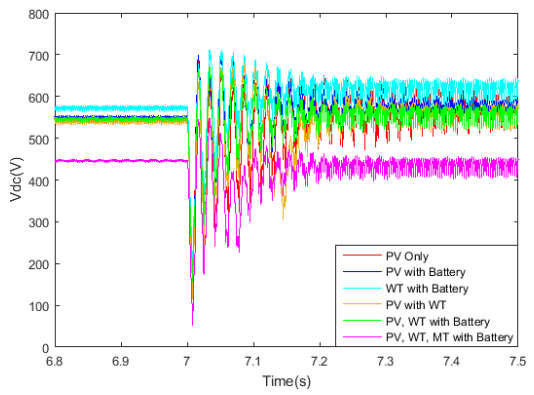

Fig. 13 DC bus voltage from islanded to grid-connected mode

\section{Active Power and Reactive Power}

The active power supplied by the microgrid during mode switching is similar to AC voltage which can be seen in Figs. 14 and 15 . However, during the reconnection to the utility grid, the microgrid active power outputs in cases A and D have more ripples. For case A, the reason is that the PV unit lacks the ability to synchronize with the utility grid quickly as it is inertia-less. For case D, a significant disturbance appears before the output power steadies (due to the effect of the wind turbine). Since the battery energy storage has the function to stabilize the DC voltage, the active power output change can be more stable in case $\mathrm{E}$.

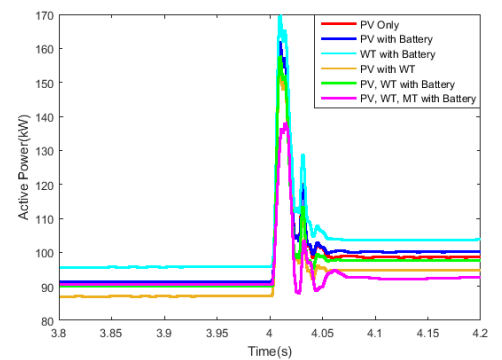

Fig. 14 Active power offered by microgrid from grid-connected to islanded mode

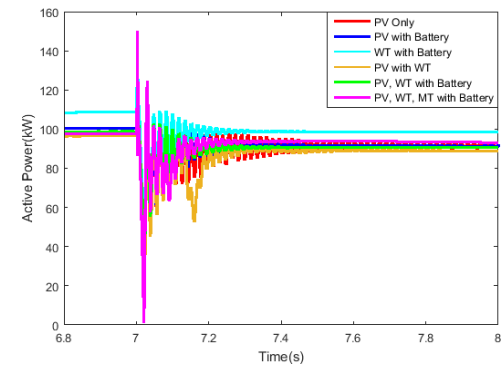

Fig. 15 Active power offered by microgrid from islanded to grid-connected mode

Figs. 16 and 17 show the reactive power generated by the microgrid during mode switching. In grid-connected mode, DER units absorb reactive power from the utility grid to regulate the voltage. However, under islanded mode, DER units supply reactive power to loads. In case $\mathrm{F}$, the reactive power required for microgrid is lower because the microturbine is able to supply reactive power under P-Q control strategy. During the transition from grid-connected to islanded mode, the reactive power output variations are quite similar in different scenarios. However, during opposite mode transition, the reactive power in case $\mathrm{D}$ and case $\mathrm{F}$ is different. As mentioned before, the WT cannot stabilize the output itself. Though the PV could offer stable power, it is still not able to eliminate the effect of the wind turbine since they share the power output equally. Therefore, the reactive power oscillates and sag during 7.1 7.2s in case D. In case F, when the microgrid is reconnected to the grid, reactive power required from the grid suddenly increase. To match this change, the reactive power offered by the micro-turbine has a sharp increment. However, due to its capacity limit, the sudden reduction of reactive power required is small which produces the sag at $7.1 \mathrm{~s}$. 


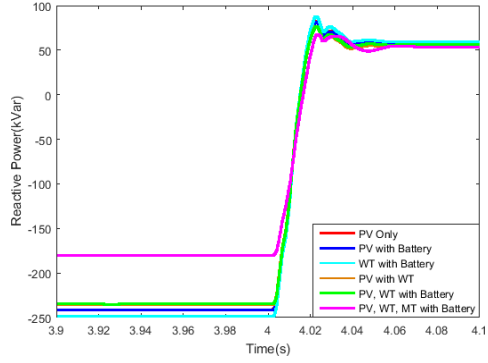

Fig. 16 Reactive power offered by Microgrid from grid-connected to islanded mode

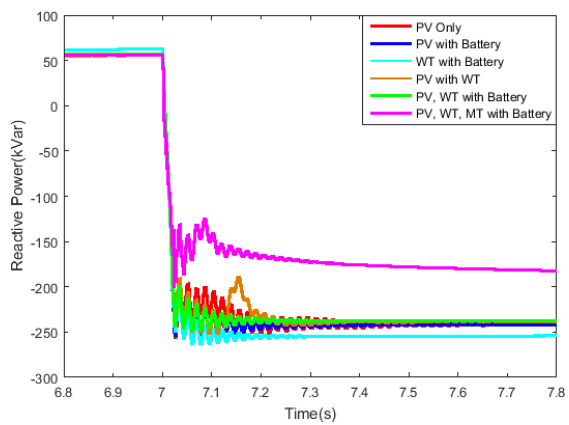

Fig. 17 Reactive power offered by microgrid from islanded to grid-connected mode

\section{CONCLUSION}

In this paper, a control strategy is proposed for a multisource hybrid AC/DC microgrid based on droop control strategy, while enabling the microgrid system to operate under both grid-connected and islanded mode. The performances of the microgrids during mode transitions, with different structures, are investigated by nonlinear simulations. Simulation results show the effectiveness of different DER units for a hybrid microgrid during mode switching. The PV unit shows a negative effect for synchronization with the utility grid. A parallel connection with other types of DER units could conquer such disadvantage. If a wind turbine is considered for connection to the DC side, necessary measures to stabilize the DC voltage should be taken into consideration. The battery performs well and enables a smooth transition of microgrid when discharging however the charging case for battery energy storage is not considered in this paper. This could be taken into consideration in the future. The micro-turbine is operating under a P-Q control strategy and has a positive effect, except it needs to be synchronized to the microgrid during transition from grid-tied to islanded mode. However, the micro-turbine should not be switched on until the microgrid is disconnected from the grid since it is mainly used as a back-up generation for the UPS. Because of its stable output and higher capacity, it should be the main resource during islanded mode which decides the microgrid frequency and voltage level. The effect of the micro-turbine dynamic effect for the microgrid will be investigated later. Moreover, according to the Griffith University microgrid system structure plan, an EV charge station will also be constructed in the future. The coordination of the complete Griffith microgrid system will be studied in future research.
APPENDIX A

TABLE I. DER Power Proportion (Approximately)

\begin{tabular}{|c|c|c|c|c|}
\hline & $\boldsymbol{P V}$ & $\boldsymbol{W T}$ & Battery & $\boldsymbol{M T}$ \\
\hline Case A & $100 \%$ & $/$ & $/$ & $/$ \\
\hline Case B & $70 \%$ & $/$ & $30 \%$ & $/$ \\
\hline Case C & $/$ & $70 \%$ & $30 \%$ & $/$ \\
\hline & $\boldsymbol{P V}$ & $\boldsymbol{W T}$ & Battery & $\boldsymbol{M T}$ \\
\hline Case D & $50 \%$ & $50 \%$ & $/$ & $/$ \\
\hline Case E & $35 \%$ & $35 \%$ & $30 \%$ & $/$ \\
\hline Case F & $25 \%$ & $25 \%$ & $30 \%$ & $20 \%$ \\
\hline
\end{tabular}

\section{REFERENCES}

[1] Huang Jiayi, Jiang Chuanwen, Xu Rong, "A review on distributed energy resources and MicroGrid," Renewable and Sustainable Energy Reviews, Volume 12, Issue 9, December 2008, Pages 2472-2483.

[2] X. Liu, P. Wang and P. C. Loh, "A Hybrid AC/DC Microgrid and Its Coordination Control," in IEEE Transactions on Smart Grid, vol. 2, no. 2, pp. 278-286, June 2011.

[3] E S N Raju P and Trapti Jain, "Hybrid AC/DC Micro Grid: An Overview," Fifth International Conference on Power and Energy Systems, October, 2013.

[4] A. Khodaei and M. Shahidehpour, "Microgrid-Based Co-Optimization of Generation and Transmission Planning in Power Systems," in IEEE Transactions on Power Systems, vol. 28, no. 2, pp. 1582-1590, May 2013.

[5] C. Ding and K. L. Lo, "Microgrid control and management of state transition period," 2012 47th International Universities Power Engineering Conference (UPEC), London, 2012, pp. 1-5.

[6] T. L. Vandoorn, B. Meersman, J. D. M. De Kooning and L. Vandevelde, "Transition From Islanded to Grid-Connected Mode of Microgrids With Voltage-Based Droop Control," in IEEE Transactions on Power Systems, vol. 28, no. 3, pp. 2545-2553, Aug. 2013.

[7] F. Ding, K. A. Loparo and C. Wang, "Modeling and simulation of gridconnected hybrid AC/DC microgrid," 2012 IEEE Power and Energy Society General Meeting, San Diego, CA, 2012, pp. 1-8.

[8] P. C. Loh and F. Blaabjerg, "Autonomous operation of hybrid microgrid with AC and DC sub-grids," Power Electronics and Applications (EPE 2011), Proceedings of the 2011-14th European Conference on, Birmingham, 2011, pp. 1-10.

[9] Hyeon-Kyun Ji, Hyeong-Jun Yoo, Thai-Thanh Nguyen, and Hak-Man Kim, "Applying Improved Droop Control to Hybrid Microgrid Control," International Journal of Control and Automation, vol. 8, no. 7 (2015), pp. 395-404.

[10] H. Xiao, A. Luo, Z. Shuai, G. Jin and Y. Huang, "An Improved Control Method for Multiple Bidirectional Power Converters in Hybrid AC/DC Microgrid," in IEEE Transactions on Smart Grid, vol. 7, no. 1, pp. 340347, Jan. 2016.

[11] Y. A. R. I. Mohamed and A. A. Radwan, "Hierarchical Control System for Robust Microgrid Operation and Seamless Mode Transfer in Active Distribution Systems," in IEEE Transactions on Smart Grid, vol. 2, no. 2, pp. 352-362, June 2011.

[12] P. Arboleya et al., "Efficient Energy Management in Smart MicroGrids: ZERO Grid Impact Buildings," in IEEE Transactions on Smart Grid, vol. 6, no. 2, pp. 1055-1063, March 2015.

[13] C. Wang et al., "A Highly Integrated and Reconfigurable Microgrid Testbed with Hybrid Distributed Energy Sources," in IEEE Transactions on Smart Grid, vol. 7, no. 1, pp. 451-459, Jan. 2016.

[14] K. De Brabandere, B. Bolsens, J. Van den Keybus, A. Woyte, J. Driesen and R. Belmans, "A Voltage and Frequency Droop Control Method for Parallel Inverters," in IEEE Transactions on Power Electronics, vol. 22, no. 4, pp. 1107-1115, July 2007.

[15] S. Golestan, M. Monfared, J. M. Guerrero and M. Joorabian, "A D-Q synchronous frame controller for single-phase inverters," Power Electronics, Drive Systems and Technologies Conference (PEDSTC), 2011 2nd, Tehran, 2011, pp. 317-323.

[16] D. N. Gaonkar and R. N. Patel, "Modeling and simulation of microturbine based distributed generation system," 2006 IEEE Power India Conference, New Delhi, 2006, pp. 5-9. 